\title{
Fingerprint imaging by scanning electrochemical microscopy
}

\author{
Meiqin Zhang, Hubert H. Girault * \\ Laboratoire d'Electrochimie Physique et Analytique, Ecole Polytechnique Fédérale de Lausanne, CH-1015 Lausanne, Switzerland
}

Received 14 March 2007; received in revised form 30 March 2007; accepted 31 March 2007

Available online 12 April 2007

\begin{abstract}
An efficient strategy for visualizing human fingerprints on a poly(vinylidene difluoride) membrane (PVDF) by scanning electrochemical microscopy (SECM) has been developed. Compared to a classical ink fingerprint image, here the ink is replaced by an aqueous solution of bovine serum albumin (BSA). After placing the "inked" finger on a PVDF membrane, the latent image is stained by silver nitrate and the fingerprint is imaged electrochemically using potassium hexachloroiridate (III) $\left(\mathrm{K}_{3} \mathrm{IrCl}_{6}\right)$ as a redox mediator. SECM images with an area of $5 \mathrm{~mm} \times 3 \mathrm{~mm}$ have been recorded with a high-resolution using a 25 - $\mu \mathrm{m}$-diameter Pt disk-shaped microelectrode. Pores in the skin (40-120 $\mu \mathrm{m}$ in diameter) and relative locations of ridges were clearly observed. The factors relevant to the quality of fingerprint images are discussed.
\end{abstract}

(C) 2007 Elsevier B.V. All rights reserved.

Keywords: Scanning electrochemical microscopy; Silver staining; Fingerprint; Imaging

\section{Introduction}

Fingerprints, impressions of the friction ridges of all or any part of a human finger, have appeared at various stages of human history, for example on ancient potteries [1-3]. Fingerprinting has often been and is still considered as one of the most widely used biometric methods. It provides a valuable physical evidence of identification, as it is among the few biometric signatures that can be truly unique and invariable for an individual. There are, in general, three forms of fingerprint evidence that may be found at crime scenes: visible (or patent) fingerprints such as those made when an ink-coated finger touches a surface and leaves prints, impression (or plastic) fingerprints, which are a mechanical impression in soft materials such as wax, and latent fingerprints, which result from a transfer of secretions from the finger to a surface. In the past, powder dusting [4], ninhydrin spraying [5], iodine fuming [6], and silver nitrate soaking [7] were the four most commonly used techniques of latent fingerprint development. These conventional techniques are quite effective in the recovery of

\footnotetext{
${ }^{*}$ Corresponding author. Tel.: +41 21693 3145; fax: +41216933667.

E-mail address: hubert.girault@epfl.ch (H.H. Girault).
}

latent fingerprints under many ordinary circumstances. However, latent fingerprints can be deposited on objects or surfaces with unique characteristics: surfaces with multicolored backgrounds such as bank notes, surfaces contaminated with blood or other body fluids, and porous surfaces such as paper. Under these conditions, traditional methods of latent fingerprint development are often ineffective.

Scanning electrochemical microscopy (SECM) [8,9], a scanning probe technique, has been successfully developed into a powerful analytical tool for the kinetic measurements of heterogeneous and homogeneous reactions [10-13], for high-resolution imaging of biomolecules immobilized onto various surfaces and biological systems [14-26], and for microfabrication [27-29]. Instrumentally, SECM comprises a microelectrode as a tip, i.e. an electrode with a micro-disk diameter of $25 \mu \mathrm{m}$ or smaller to electrochemically detect a specific redox species near the surface of a substrate, a high-precision position controller, and a bipotentiostat. In SECM experiments, the microelectrode is brought to the vicinity of the substrate surface, where the electrochemical response of the tip is recorded as a function of the lateral tip position $(x, y)$ for imaging. Compared with other scanning probe microscopic techniques, SECM possesses some unique features. For example, it is 
capable of imaging chemical or biochemical activities present at a substrate surface and can image samples with relatively large surface areas (a few square centimeters). The latter feature makes SECM particularly advantageous over other scanning probe techniques for the imaging of latent fingerprints on a PVDF membrane. Moreover, SECM also has a well-developed quantitative theory.

We had previously shown that the SECM approach could be used to image silver or copper stained proteins $[30,31]$. These reports demonstrated the feasibility of using SECM for visualizing protein spots on a membrane surface. More recently, we improved this methodology as a highly sensitive tool of imaging proteins separated by gel electrophoresis, electroblotted on PVDF membranes and then silver stained [32]. Here, the technique is used to visualize latent fingerprints on a poly(vinylidene difluoride) (PVDF) membrane using SECM imaging of silver stained proteins. The fingerprints were formed on the membrane by microcontact printing ( $\mu \mathrm{CP})[33,34]$, using a human finger rather than a poly(dimethylsiloxane) stamp. An aqueous solution of bovine serum albumin (BSA) at $1 \mathrm{mg} / \mathrm{mL}$ concentration was used as the "ink". This methodology takes advantage of the high sensitivity of SECM towards the small variation of electrochemical properties at the substrate surface. It is also applicable to bloody latent fingerprints. The SECM images of the human fingerprints are of comparable or better quality than those obtained by conventional optical methods.

\section{Experimental}

\subsection{Chemicals and materials}

All chemicals were used as received. Methanol (Merck), sodium acetate anhydrous ( $>99 \%$, Fluka), sodium thiosulfate pentahydrate $\left(\mathrm{Na}_{2} \mathrm{~S}_{2} \mathrm{O}_{3} \cdot 5 \mathrm{H}_{2} \mathrm{O},>99.5 \%\right.$, Fluka), sodium carbonate anhydrous $\left(\mathrm{Na}_{2} \mathrm{CO}_{3},>99.5 \%\right.$, Fluka), formaldehyde ( $37 \mathrm{wt}^{\%} \%$ solution in water, Aldrich), silver nitrate $\left(\mathrm{AgNO}_{3},>99.5 \%\right.$, Fluka), ammonium nitrate $\left(\mathrm{NH}_{4} \mathrm{NO}_{3}, \geqslant 99.5 \%\right.$, Fluka), tungstosilicic acid hydrate $(>99.9 \%$, Aldrich), acetic acid ( $>99.8 \%$, Fluka), potassium hexachloroiridate (III) $\left(\mathrm{K}_{3} \mathrm{IrCl}_{6}\right.$, Aldrich), potassium nitrate $\left(\mathrm{KNO}_{3},>99 \%\right.$, Fluka). Immun-Blot PVDF membranes for protein blotting $(0.2 \mu \mathrm{m})$ were purchased from Bio-Rad (Hercules, CA, USA). Water was deionized to a conductivity of $18.2 \mu \mathrm{S} \mathrm{cm} \mathrm{cm}^{-1}$ using a Milli Q plus 185 from Millipore. Bovine serum albumin (BSA, $\geqslant 98 \%$ ) was purchased from Sigma. Alumina $1 \mu \mathrm{m},(0.3$ and $0.05 \mu \mathrm{m}$, $)$ and Mastertex polishing cloths from Buehler were employed to polish the $25 \mu \mathrm{m}$-diameter disk shaped microelectrode.

\subsection{Preparation of human fingerprints on the PVDF membrane}

The fingerprints were formed by $\mu \mathrm{CP}$ of an "anonymous human finger" inked by a BSA solution on a PVDF membrane. The hydrophobicity of a PVDF membrane makes it an ideal support for binding proteins. Because of the hydrophobic nature of PVDF, it was first wetted in methanol for 1-3 s and then immersed in water for 1-2 min to elute the methanol when used as the substrate for fingerprints. (Caution! Once the membrane has been wetted with water, do not allow it to dry until the proteins have been transferred to it.) Prior to the $\mu \mathrm{CP}$ process, the finger of the anonymous volunteer was thoroughly cleaned with deionized water and dried with $\mathrm{N}_{2}$. After "inking" the finger for $2 \mathrm{~min}$ with a $1 \mathrm{mg} / \mathrm{mL}$ BSA solution and allowing it to dry under a $\mathrm{N}_{2}$ stream, it was then gently pressed onto the pre-wetted PVDF membrane substrate for $30 \mathrm{~s}$. After removal of the finger, the protein marked membrane was submerged in a $1 \%$ sodium acetate solution for the subsequent silver staining process.

The optical fingerprint on the PVDF membrane obtained by inking the same finger using a standard writing ink was prepared in the same manner. After carefully cleaning the finger with deionized water and drying it with $\mathrm{N}_{2}$, it was inked for $10 \mathrm{~s}$, and then placed into conformal contact with a pre-wet PVDF membrane substrate for $10 \mathrm{~s}$. After removing the finger, the fingerprint on the membrane substrate was imaged by an optical laser-scanner (HP Scanjet 4890).

\subsection{Silver staining procedure of protein fingerprints on the PVDF membrane}

The staining process followed for the procedure was that outlined by Sørensen et al. [35]. The protein fingerprinted sample was washed $15 \mathrm{~min}$ in $1 \%$ sodium acetate. It was then rinsed twice with water for $1 \mathrm{~min}$. The sample was sensitized $15 \mathrm{~min}$ in $0.1 \%$ sodium thiosulfate, and it was then rinsed twice with deionized water for $1 \mathrm{~min}$. After rinsing, the sample was submerged in a Gallyas staining solution made from two stock solutions (A and B), which can be stored for months at room temperature when protected against the light: (A) $5 \mathrm{~g}$ sodium carbonate dissolved in $100 \mathrm{~mL}$ of water; (B) $0.2 \mathrm{~g}$ ammonium nitrate, $0.2 \mathrm{~g}$ silver nitrate, $1 \mathrm{~g}$ tungstosilicic acid, and $0.5 \mathrm{~mL} 37 \%$ formaldehyde were added to $100 \mathrm{~mL}$ of water. The Gallyas stock A and stock B were mixed 1:1 under intensive shaking and the sample developed in this for $10 \mathrm{~min}$. The development was stopped in $10 \%$ acetic acid for $5 \mathrm{~min}$, and then the sample was dried for further characterization by SECM (CH Instruments Model CHI 900, USA).

\subsection{SECM measurements}

A three-electrode setup was employed with a $25-\mu \mathrm{m}$ diameter Pt disk shaped microelectrode as the amperometric SECM tip as schematically presented in Fig. 1. The counter and quasi-reference electrodes were a $\mathrm{Pt}$ wire and a silver wire, respectively. The protein fingerprinted membrane was fixed on a microscope glass slide. This cell assembly was secured onto a platform which includes three 


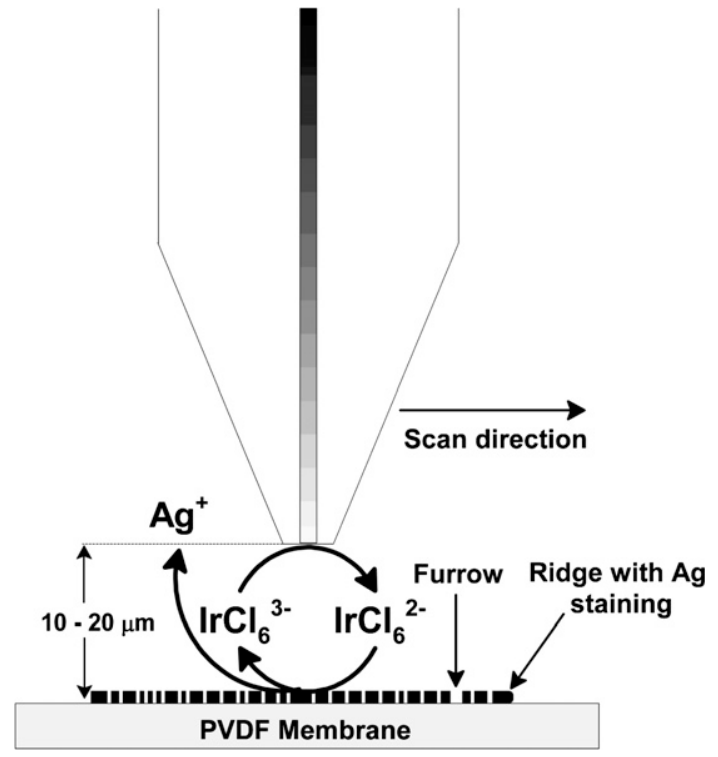

Fig. 1. Schematic (not to scale) representation of the operating principle for SECM imaging of silver stained fingerprints on the PVDF membrane using $\mathrm{IrCl}_{6}^{3-}$ oxidation ( $E_{\text {tip }}=0.8 \mathrm{~V}$ vs. $\left.\mathrm{Ag} \mathrm{QRE}\right)$ at the tip.

screws for leveling the substrate surface. The Pt microelectrode is prepared from a $25-\mu \mathrm{m}$-diameter Pt wire using the procedures described previously [36]. A Laborlux D optical microscope (Leitz, Germany) is used to check the quality of the prepared tip. The microelectrode is polished, washed with distilled water and acetone, and dried prior to each measurement. The RG value of the microelectrode ( $\mathrm{RG}=R_{\mathrm{g}} / r$, where $R_{\mathrm{g}}$ is the radius of the insulating glass) is about 3.5. Feedback images of fingerprints were performed in $2 \mathrm{mM} \mathrm{K}_{3} \mathrm{IrCl}_{6}+0.1 \mathrm{M} \mathrm{KNO}_{3}$ solution at a tip potential of $E_{\text {tip }}=0.8 \mathrm{~V}$ (vs. Ag QRE) while the sample was at open circuit potential (OCP). The tip was brought to ca. $15 \mu \mathrm{m}$ above the fingerprinted substrate surface by monitoring the steady-state oxidation current of $\mathrm{IrCl}_{6}^{3-}$. Although this SECM instrument is in principle capable of scanning an area of $5 \mathrm{~cm} \times 5 \mathrm{~cm}$, with the aim of illustration, an image of smaller area (e.g., $0.5 \mathrm{~cm} \times 0.3 \mathrm{~cm}$ ) is shown in this work. All of the SECM experiments were carried out at room temperature $\left(22 \pm 2{ }^{\circ} \mathrm{C}\right)$.

\section{Results and discussion}

In the amperometric feedback mode of SECM measurements, the tip signal used for imaging is a faradaic current, $i$, originating from the oxidation of the mediator $\mathrm{IrCl}_{6}^{3-}$ added to the electrolyte solution. With the tip kept at a constant potential ( $0.8 \mathrm{~V}$ vs. Ag QRE) high enough to oxidize $\mathrm{IrCl}_{6}^{3-}$ and placed in the bulk solution far above the surface of the fingerprinted substrate, a diffusion-limited steady-state current $\left(i_{\infty}\right)$ is observed. When the tip is brought close to ridges of the silver-stained fingerprint sample, electrochemical recycling of the $\mathrm{IrCl}_{6}^{3-}$ becomes possible by the following heterogeneous bimolecular electron transfer reaction between $\mathrm{IrCl}_{6}^{2-}$ and silver nanoparticles [32]:
$\mathrm{Ag}(\mathrm{s})+\mathrm{IrCl}_{6}^{2-}(\mathrm{aq}) \stackrel{k_{\mathrm{ET}}}{\rightarrow} \mathrm{Ag}^{+}(\mathrm{aq})+\mathrm{IrCl}_{6}^{3-}(\mathrm{aq})$

This process leads to an increase in tip current (positive feedback effect). On the other hand, the tip current decreases monotonically with the tip approaching the blank or furrows of the substrate because $\mathrm{IrCl}_{6}^{3-}$ diffusion hindrance (negative feedback effect). Changes in the tip current during lateral scans used for "chemical imaging" reflect the topology of the silver-stained fingerprint on the surface. The approach curve was carried out with $2 \mathrm{mM}$ $\mathrm{K}_{3} \mathrm{IrCl}_{6}+0.1 \mathrm{M} \mathrm{KNO}_{3}$ solution at a potential of $E_{\text {tip }}=0.8 \mathrm{~V}$ (vs. Ag QRE) on a blank area of the surface, and was stopped when the tip touched the membrane. The tip was then retracted about $15 \mu \mathrm{m}$ and coarsely positioned above the surface of fingerprints for imaging.

The feedback mode of SECM can be observed when the tip is close to the surface usually at a distance less than twice the tip radius. Here, the tip-substrate distance was kept to about $15 \mu \mathrm{m}$ when scanning the tip over a large area (e.g., $5 \mathrm{~mm} \times 3 \mathrm{~mm}$ ) of fingerprinted surface. The tip is moved rather than the substrate using piezoelectric and "inchworm" motors. The spatial resolution of the motors is high $(0.02-0.1 \mu \mathrm{m})$ in comparison with the size of the standard SECM tips $(2-25 \mu \mathrm{m}$ diameter). Theoretically, the spatial resolution of SECM is mainly governed by the tip radius $r$, i.e., a smaller tip offers a higher spatial resolution, as the tip-substrate distance is reduced. This short distance is difficult to maintain when imaging large areas that may be tilted with respect to the traveling tip and tip crash can occur. With the objective of imaging a relatively large area $(5 \mathrm{~mm} \times 3 \mathrm{~mm})$ of fingerprinted surface with a good spatial resolution, we chose a $25 \mu \mathrm{m}$-diameter tip and the sample surface was adjusted manually. Data from line scan experiments in the $X$ direction performed on a finger-

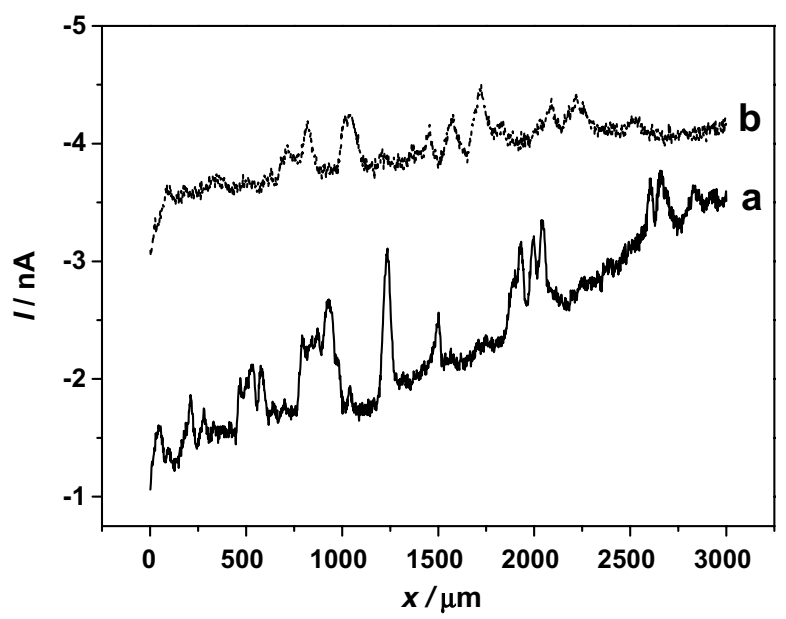

Fig. 2. Constant height current response obtained in feedback mode with $2 \mathrm{mM}$ of the mediator $\mathrm{IrCl}_{6}^{3-}$ (in $0.1 \mathrm{M} \mathrm{KNO}_{3}$ ) by scanning at $\mathrm{Pt}$ microelectrode $(25 \mu \mathrm{m}$ diameter, $\mathrm{RG}=3.5)$ in the $X$ direction above the surface of fingerprints. Solid line (a) surface tilted relative to the tip scan; short dash line (b) relatively flat substrate surface. Measuring conditions: $E_{\text {tip }}=0.8 \mathrm{~V}$ vs. Ag QRE, a tip-substrate distance of approximately $15 \mu \mathrm{m}$ and a lateral scan rate of $2 \mu \mathrm{m}$ each $0.05 \mathrm{~s}$. 
printed surface with two different tilts are illustrated in Fig. 2. The solid line (a) shows very big variations from $1.2 \times 10^{-9} \mathrm{~A}$ to $3.5 \times 10^{-9} \mathrm{~A}$ in the baseline of tip current response when scanning over a range of $3 \mathrm{~mm}$, which indicates the effect of the tip-substrate distance $d$ on the tip current, i.e., a higher current value in the baseline means a larger $d$ from the negative feedback degrading the image quality. After carefully adjusting the substrate tilt, very small variations only from $3.5 \times 10^{-9} \mathrm{~A}$ to $4.0 \times 10^{-9} \mathrm{~A}$ in the tip current baseline are observed as shown by the short dash line (b). It suggests the $X$ line of tip scanning is almost parallel to the fingerprinted surface. Of course, better results would be obtained using a shear-force compensation module or other systems able to compensate the tilt of the sample.

An optical laser-scanner image of an inked fingerprint on the PVDF membrane using a classical writing ink is illustrated in Fig. 3a. The black and white lines correspond to the ridges and furrows, respectively. According to a classification system known as the Henry-Galton system [37], loops, whorls, and arches are the basic classes of fingerprint patterns. The one shown in Fig. 3a belongs to the whorls, which constitute $25-35 \%$ of the patterns encountered [38]. We can see that some of the ridges make a turn through at least one circuit. For comparison, a high-resolution SECM image $(5 \mathrm{~mm} \times 3 \mathrm{~mm})$ of the silver stained fingerprint on the PVDF membrane is shown in Fig. 3b, which

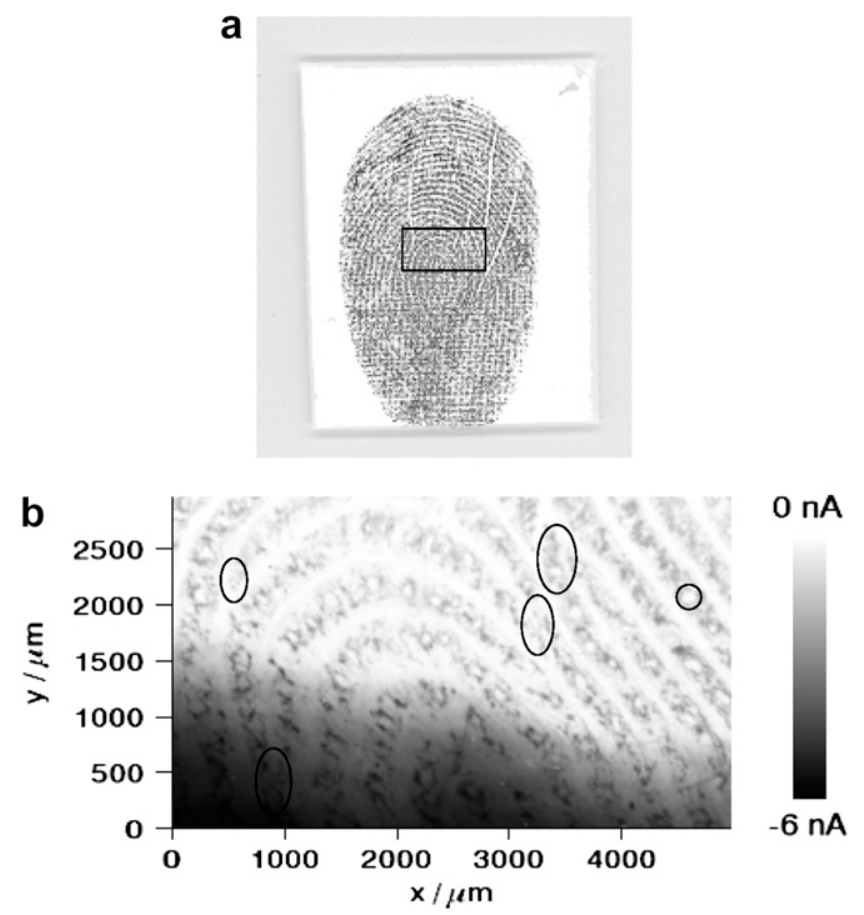

Fig. 3. (a) Optical laser-scanner image of a classical writing ink fingerprint on a PVDF membrane; (b) A SECM image $(5 \mathrm{~mm} \times 3 \mathrm{~mm})$ of the silver stained fingerprints on the PVDF membrane. The image area corresponds approximately to the rectangle region marked (inside black line) in (a). Pore shape shown in the circle. The ovals represent the characteristic points. Measuring conditions: $2 \mathrm{mM} \mathrm{K}_{3} \mathrm{IrCl}_{6}$ in $0.1 \mathrm{M} \mathrm{KNO}_{3}, E_{\text {tip }}=0.8 \mathrm{~V}$ vs. Ag QRE, a tip-substrate distance of approximately $15 \mu \mathrm{m}$ and a lateral scan rate of $20 \mu \mathrm{m}$ each $0.2 \mathrm{~s}$. needed about $6 \mathrm{~h} 15 \mathrm{~min}$ with a fast scanning speed to ensure the compatibility of the system with large surface scanning. In this case, convective and non-steady-state diffusion transient effects may have a little influence on the response [39]. The image area corresponds approximately to the rectangle region marked (inside black line) in Fig. 3a. The changes observed in SECM-generated data are mainly due to variations in the electrochemical reactivity between regions that are covered with silver-stained proteins and regions that are not except that a higher background current is presented in the region of $0 \leqslant y / \mathrm{mm} \leqslant 1$. In Fig. 3b, a dark color has been used to emphasize the regions of significant positive feedback. The dark regions correspond to the ridges because the stained proteins were transferred from the ridges of the finger to the PVDF membrane substrate. The positive feedback is due to the regeneration of $\mathrm{IrCl}_{6}^{3-}$ by the heterogeneous bimolecular electron transfer described in reaction (1). Inversely, the furrows of the finger cannot transfer proteins onto the PVDF membrane surface, so $\mathrm{IrCl}_{6}^{3-}$ cannot be recycled. The insulating nature of PVDF membrane blocks some of the diffusion of $\mathrm{IrCl}_{6}^{3-}$ to the tip surface, which leads to a lower tip current as shown in the light regions of Fig. $3 \mathrm{~b}$.

The present technique differs from the classical silver nitrate method used for developing latent fingerprints where silver nitrate is used to react with the chloride present in the fingerprint deposit residue [7]. The product of this reaction, silver chloride, rapidly turns back on exposure to light for detection. However, silver nitrate reagent does not work well with the latent fingerprints exposed to high humidity because the chloride in the deposit migrates by diffusion.

A significant feature of this proposed technique is the ability of high-resolution image for pore shape (marked with circle in Fig. 3b). Champod et al. describe three general levels of information that can be distinguished in fingerprints [2]: the first level involves the overall pattern of the papillary ridges, yet there exist many variations and sub-patterns. The second level information refers to the major deviations of the ridge paths from general patterns (marked with oval in Fig. 3b), for example hook, ending, bifurcation, ridge divergence, island, enclosure, short independent ridge, and crossover. The third level of information refers to the shape and relative position of the pores of each part of a ridge. For the moment, the first and second levels are very important for the verification of a person's identity. Comparatively little research, however, has been done on the third level information because the quality of the fingerprints is often not good enough for determining a match based on pore or ridge shape. As can be seen from Fig. 3b, pores $(40-120 \mu \mathrm{m}$ in diameter) and relative locations of ridges were clearly imaged. If decreasing the size of the SECM tip (e.g., $<2 \mu \mathrm{m}$ diameter), the quality of images will be further improved. Therefore, SECM image of fingerprints is a potential tool for obtaining the third level information to support and confirm a human identification. 
In addition, for successfully developing bloody latent fingerprints, in the past there are two general categories of chemical reagents [1]: one category of reagents is those chemicals that react with the heme moiety of the hemoglobin molecule of the red blood cells in blood, such as benzidine, tetramethylbenzidine, o-tolidine, phenolphthalein, and leucomalachite green; the other category of reagents is general protein stains, commonly used protein dyes such as amido black, ninhydrin, crystal violet, and Commassie blue. As it is well-known, silver staining is a highly sensitive technique $\left(0.1 \mathrm{ng} / \mathrm{mm}^{2}\right)$ for detecting proteins [30,32,35], so the present imaging strategy with SECM should also be applicable for the analysis of bloody latent fingerprints.

\section{Conclusions}

A new approach for imaging fingerprints on PVDF membranes is presented: fingerprints were first obtained on the substrate surface by $\mu \mathrm{CP}$ method using a protein solution as "ink", and then stained with silver nanoparticles which were chemically generated from the reduction of silver nitrate by formaldehyde. Finally, the fingerprinted surface was scanned by the tip of SECM, with $\mathrm{IrCl}_{6}^{3-}$ acting as the redox mediator to detect silver dissolution in positive feedback effect. A high-resolution SECM image $(5 \mathrm{~mm} \times 3 \mathrm{~mm})$ of fingerprints on the PVDF membrane was achieved, which can provide the third level valuable information for confirming an identification. The quality of fingerprints imaged by SECM is already comparable with state-of-the-art optical detection techniques. In addition, it is also compatible with the previously detecting techniques of bloody latent fingerprints. Work is in progress to extend this approach to image latent fingerprints enhanced by a process known as "MultiMetal-Deposition" (MMD) where gold nanoparticles adhere to the fingerprint residue and catalyze the precipitation of metallic silver from the silver physical developer solution [40-42].

\section{Acknowledgements}

This work was supported by Ecole Polytechnique Fédérale de Lausanne. The authors are grateful to Prof. C. Champod from the School of Criminal Sciences and institute of forensic sciences, University of Lausanne and Fernando Cortes Salazar (LEPA, EPFL) for helpful discussions. The technical assistance by Valérie Devaud is also acknowledged.

\section{References}

[1] H.C. Lee, R.E. Gaensslen (Eds.), Advances in Fingerprint Technology, second ed., CRC Press, Boca Raton, 2001.

[2] C. Champod, C. Lennard, P. Margot, M. Stoilovic (Eds.), Fingerprints and other Ridge Skin Impressions, CRC Press, Boca Raton, 2004.

[3] P. Voss-de Hann, Contemp. Phys. 47 (2006) 209.

[4] J.D. James, C.A. Pounds, M. Phil, B. Wilshire, J. Forensic Sci. 36 (1991) 1368.
[5] S. Oden, B. von Hofsten, Nature 173 (1954) 449.

[6] M.A. Feldman, C.E. Meloan, J.L. Lambert, J. Forensic Sci. 27 (1982) 806.

[7] Y. Mikami, T. Itasaka, M. Matsumoto, C. Okamoto, Kagaku To Sosa 12 (1959) 518.

[8] A.J. Bard, F.-R.F. Fan, M.V. Mirkin, in: A.J. Bard (Ed.), Scanning Electrochemical Microscopy, vol. 18, Marcel Dekker, New York, 1994, pp. 244-391.

[9] A.J. Bard, M.V. Mirkin (Eds.), Scanning Electrochemical Microscopy, Marcel Dekker, New York, 2001.

[10] M.V. Mirkin, T.C. Richards, A.J. Bard, J. Phys. Chem. 97 (1993) 7672.

[11] A.L. Barker, M. Gonsalves, J.V. Macphercon, C.J. Slevin, P.R. Unwin, Anal. Chim. Acta 385 (1999) 223.

[12] Z. Zhang, Y. Yuan, P. Sun, B. Su, J. Guo, Y. Shao, H.H. Girault, J. Phys. Chem. B 106 (2002) 6713.

[13] P. Sun, F. Li, Y. Chen, M. Zhang, Z. Zhang, Z. Gao, Y. Shao, J. Am. Chem. Soc. 125 (2003) 9600.

[14] F.-R.F. Fan, A.J. Bard, Proc. Natl. Acad. Sci. USA 96 (1999) 14222.

[15] B.R. Horrocks, D. Schmidtke, A. Heller, A.J. Bard, Anal. Chem. 65 (1993) 3605.

[16] D.T. Pierce, P.R. Unwin, A.J. Bard, Anal. Chem. 64 (1992) 1795.

[17] H. Shiku, T. Akeda, H. Yamada, T. Matsue, I. Uchida, Anal. Chem. 67 (1995) 312.

[18] H. Shiku, T. Matsue, I. Uchida, Anal. Chem. 68 (1996) 1276.

[19] G. Wittstock, K.-J. Yu, H.B. Halsall, T.H. Ridgeway, W.R. Heineman, Anal. Chem. 67 (1995) 3578.

[20] G. Wittstock, W. Schuhmann, Anal. Chem. 69 (1997) 5059.

[21] J.V. Macpherson, P.R. Unwin, Anal. Chem. 73 (2001) 550.

[22] J. Wang, F.Y. Song, F.M. Zhou, Langmuir 18 (2002) 6653.

[23] L.P. Bauermann, W. Schuhmann, A. Schulte, Phys. Chem. Chem. Phys. 6 (2004) 4003.

[24] H. Yamada, H. Fukumoto, T. Yokoyama, T. Koike, Anal. Chem. 77 (2004) 1785.

[25] A. Amemiya, J.D. Guo, H. Xiong, D.A. Gross, Anal. Bioanal. Chem. 386 (2006) 458.

[26] B. Liu, S.A. Rotenberg, M.V. Mirkin, Proc. Natl. Acad. Sci. USA 97 (2000) 9855.

[27] Y. Yatziv, I. Turyan, D. Mandler, J. Am. Chem. Soc. 124 (2002) 5618.

[28] I. Turyan, M. Etienne, D. Mandler, W. Schuhmann, Electroanalysis 17 (2005) 5.

[29] A.K. Neufeld, A.P. O'Mullane, A.M. Bond, J. Am. Chem. Soc. 127 (2005) 13846.

[30] M. Carano, N. Lion, J.-P. Abid, H.H. Girault, Electrochem. Commun. 6 (2004) 1217.

[31] M. Carano, N. Lion, H.H. Girault, J. Electroanal. Chem. 599 (2007) 349.

[32] M.Q. Zhang, G. Wittstock, Y.H. Shao, H.H. Girault, Anal. Chem., accepted for publication.

[33] A. Kumar, G.M. Whitesides, Appl. Phys. Lett. 63 (1993) 2002.

[34] Y. Xia, X.M. Zhao, G.M. Whitesides, Mircoelectron. Eng. 32 (1996) 255.

[35] B.K. Sørensen, P. Højrup, E. Østergård, C.S. Jørgensen, J. Enghild, L.R. Ryder, G. Houen, Anal. Biochem. 304 (2002) 33.

[36] C. Lee, C.J. Miller, A.J. Bard, Anal. Chem. 63 (1991) 78.

[37] E.R. Henry, Classification and Uses of Finger Prints, fourth ed., Georges Routedge, London, 1900.

[38] $<$ http://www.policensw.com/info/fingerprints/finger07.html $>$.

[39] C. Lefrou, J. Electroanal. Chem. 601 (2007) 394.

[40] G. Saunders, International Association for Identification, in: 74th Annual Educational Conference, Pensacola, USA, June, 1989.

[41] B. Schnetz, P. Margot, Forensic Sci. Int. 118 (2001) 21.

[42] M. Sametband, I. Shweky, U. Banin, D. Mandler, J. Almog, Chem. Commun. 11 (2007) 1142. 